\title{
Absolut messender Winkelsensor auf Basis optischer Interferometrie
}

\author{
Erik Oertel ${ }^{1}$, Vinzenz Ullmann ${ }^{1}$, Eberhard Manske ${ }^{1}$ \\ ${ }^{1}$ Institut für Prozessmess- und Sensortechnik, Technische Universität IImenau, PF 100565,
}

D-98694 IImenau

\begin{abstract}
Zusammenfassung
Zur absoluten, hochauflösenden Winkelmessung reflektierender Oberflächen werden nach dem derzeitigen Stand der Technik Autokollimationsfernrohre eingesetzt. Die mit diesem Prinzip erreichbaren Eigenschaften in Bezug auf die Sensorgröße, Auflösung und Messunsicherheit sind für Anwendungen mit hohen Anforderungen nicht mehr ausreichend. Aus diesem Grund wurde ein neuartiges Verfahren zur absoluten Winkelmessung entwickelt. Es basiert auf einem Kösters-Prisma und optischer Interferometrie. Das Interferenzsignal wird über eine CMOS-Matrix erfasst und durch einen Algorithmus ausgewertet. Als Signal für die Winkellage dient die Neigung und Streifenbreite der Interferenzstreifen. Bei ersten messtechnischen Untersuchungen konnte mit diesem Verfahren bereits eine Auflösung von unter 5 nrad und ein Messbereich von mehr als 5 mrad erreicht werden. Im Rahmen dieses Beitrages wird das neue Sensorkonzept vorgestellt. Das beinhaltet den optischen Sensoraufbau, die Strategie zur Signalauswertung und erste messtechnische Untersuchungen.
\end{abstract}

Keywords: Winkelsensor, Interferometer, CMOS-Matrix, digitale Bildverarbeitung, Kösters-Prisma

\section{Einleitung}

Zur hochauflösenden, absoluten Winkelmessung von verspiegelten Oberflächen kommen nach dem derzeitigen Stand der Technik primär Autokollimationsfernrohre (AKFs) zum Einsatz . Bei diesen Messgeräten wird die Empfindlichkeit - und damit die erreichbare Auflösung - maßgeblich durch die Brennweite des Kollimators bestimmt. Die mit diesem Prinzip erreichbaren Eigenschaften in Bezug auf die Sensorgröße, Auflösung und Messunsicherheit sind für Anwendungen mit hohen Anforderungen nicht mehr ausreichend. Als Alternative werden nach dem derzeitigen Stand mehrstrahlige Interferometer eingesetzt. Diese Geräte führen die Winkelmessung auf eine Differenzlängenmessung zurück und erreichen die Anforderungen in den genannten Eigenschaften. Im Gegensatz zu AKFs ist mit mehrstrahligen Interferometern aber nur eine relative Winkelmessung möglich. Daher können diese Interferometer die Funktion von AKFs nicht vollständig ersetzen.

Am Institut für Prozessmess- und Sensortechnik der TU IImenau wurde deshalb ein neuartiges interferometrisches Verfahren zur absoluten Winkelmessung entwickelt und untersucht [1] , [2] (Abbildung 1).
In diesem wird ein Kösters-Prisma durch einen divergenten, kurz-kohärenten Laserstrahl beleuchtet und an dem halbdurchlässigen Strahlteiler aufgeteilt. Beide Strahlen werden an dem zu vermessenden Spiegel reflektiert und interferieren anschließend auf der gegenüberliegenden Seite. Das dadurch entstehende Interferenzbild wird durch eine CMOS-Matrix erfasst. Als Signal für den Spiegelneigungswinkel $\varphi_{x}$ wird der Winkel der Interferenzstreifen $\Psi$ genutzt. Dieser bietet eine absolute Referenz, weil jedem Winkel $\Psi$ exakt ein Spiegelneigungswinkel $\varphi_{x}$ zugeordnet werden kann (Abbildung 2).

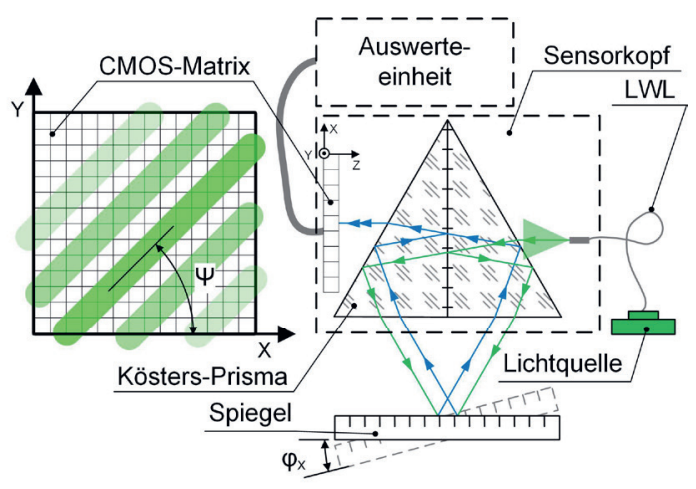

Abb. 1: Das in [1] und [2] vorgestellte Sensorkonzept zur Winkelmessung 
Anhand eines experimentellen Aufbaus konnte in [1] und [2] nachgewiesen werden, dass mit diesem Verfahren hochauflösende absolute Winkelmessungen möglich sind. Die Kennlinie wurde durch eine Vergleichsmessung mit einem AKF ermittelt und kann durch GI. (1) beschrieben werden.

$\varphi_{\mathrm{x}}=\mathrm{b} \tan (\Psi)$

Der Faktor b wird von Fertigungsabweichungen der einzelnen Kösters-Prismen beeinflusst und muss dementsprechend für jeden Sensor separat kalibriert werden. In experimentellen Untersuchungen wurden darüber hinaus weitere Einflussfaktoren auf den Parameter b ermittelt. Das betrifft beispielsweise den Abstand des Spiegels vom Kösters-Prisma. Weil der quantitative Wert von b durch die zusätzlichen Einflussgrößen - ohne weitere Maßnahmen - mit einer hohen Unsicherheit verbunden ist, ist eine Anwendung des vorgestellten Sensors lediglich als Nullpunktindikator denkbar. Für diesen Anwendungsfall ist nur die Auflösung und Stabilität von dem Winkel $\Psi$ in der Nullage für die erreichbare Messunsicherheit limitierend.

Im Bezug auf die Auflösung und Stabilität konnten gegenüber AKFs mit Brennweiten von $300 \mathrm{~mm}$ bereits hervorragende Eigenschaften realisiert werden. Um das Prinzip für absolute Messungen außerhalb der Nullage zu Nutzen, ist eine Reduktion der limitierenden Einflussgrößen notwendig.

Mit dieser Ziellstellung wurde das Verfahren weiterentwickelt und untersucht. Im Rahmen dieses Beitrags werden die wesentlichen Erkenntnisse und Ergebnisse dieser Untersuchungen vorgestellt. Das betrifft Änderungen am optischen Sensoraufbau und der Signalauswertung.

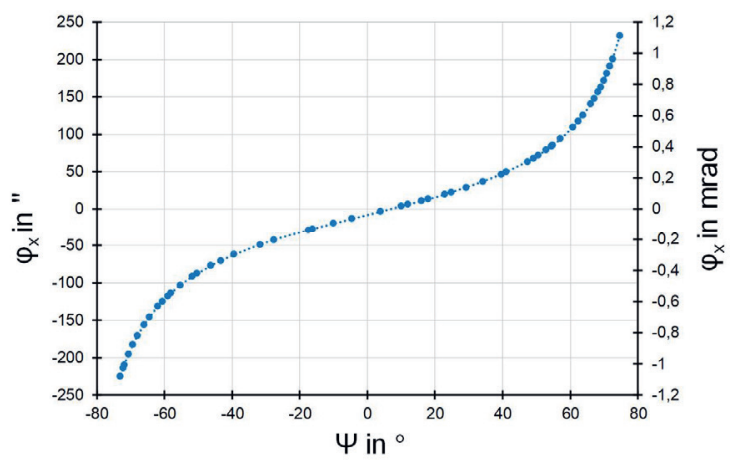

Abb. 2: Abhängigkeit zwischen dem Neigungswinkel des Spiegels und der Interferenzstreifen

\section{Grundlagen}

Die Abstandsabhängigkeit des in [1] und [2] vorgestellten Sensors wird primär durch die Nutzung von divergentem Licht verursacht und kann durch den Übergang zu kollimiertem Licht reduziert werden. In der bisherigen Konfiguration des Strahlengangs konnte in diesem Fall allerdings keine Neigungsänderung der Interferenzstreifen beobachtet werden.

Unter der Annahme von zwei ideal kollimierten Strahlen (ebene Wellenfronten), kann der Zusammenhang zwischen dem Winkelversatz der Strahlen (Abbildung 3) und dem resultierende Interferenzbild (Abbildung 4) durch GI. (2), GI. (3) und GI. (4) beschrieben werden [3],[4].

$\Psi_{\mathrm{x}}=\frac{\lambda}{\lambda^{\prime}} \sin (\Psi)$

$\Psi_{\mathrm{y}}=\frac{\lambda}{\lambda^{\prime}} \cos (\Psi)$

$\Psi=\arctan \left(\frac{\Psi_{\mathrm{x}}}{\Psi_{\mathrm{y}}}\right)$

Die Gleichungen gelten nur näherungsweise für einen geringen Winkelversatz zwischen den Strahlen $\left(\Psi_{\mathrm{x}}\right.$ und $\left.\Psi_{\mathrm{y}}\right)$, weil innen die Linearisierung trigonometrischer Funktionen zugrunde liegt. Der Parameter $\lambda$ entspricht der Wellenlänge des Laserlichts.

Bei dem vorgestellten Sensorprinzip aus Abbildung 1 wird der Spiegelneigungswinkel $\varphi_{x}$ auf einen Winkelversatz zwischen den Strahlen $\Psi_{\mathrm{x}}$ in der X-Z-Ebene übertragen. Aufgrund der Reflexion beider Strahlen am Spiegel gilt der Zusammenhang aus GI. (5) [5].

$\Psi_{\mathrm{x}}=4 \varphi_{\mathrm{x}}$

Somit ist es ausgehend von GI. (2) und GI. (5) möglich die absolute Winkelmessung für kleine Winkel auf eine Erfassung der Streifenbreite $\lambda^{\prime}$ und des Neigungswinkels $\Psi$ der Interferenzstreifen zurückzuführen. Dafür ist eine entsprechende Signalauswertung notwendig, welche im nächsten Abschnitt näher diskutiert wird.
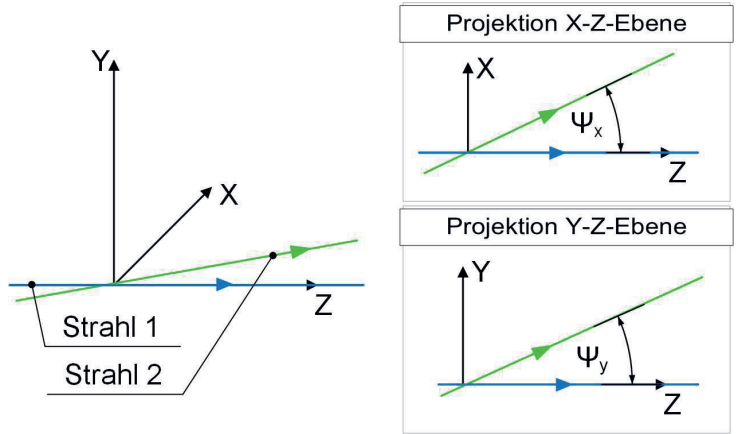

Abb. 3: Winkelversatz zwischen den interferierenden Interferenzstreifen 


\section{Strategie zur Signalauswertung}

Eine verhältnismäßig einfache Auswertung ist möglich, wenn anstatt der Streifenbreite $\lambda^{\prime}$ die auf die Spalten und Zeilen projizierten Streifenbreiten $\lambda_{y}^{\prime}$ und $\lambda_{x}{ }_{x}$ erfasst werden. In diesem Fall kann auf die aufwendigen Methoden der digitalen Bildverarbeitung verzichtet werden, indem jedes Spalten- und Zeilensignal einzeln ausgewertet wird. Die dafür geltenden Zusammenhänge ergeben sich aus den GI. (2), GI. (3) und Abbildung 4.

$\Psi_{\mathrm{x}}=\frac{\lambda}{\lambda_{y}^{\prime}} \tan (\Psi)=\frac{\lambda}{\lambda_{x}^{\prime}}$

$\Psi_{\mathrm{y}}=\frac{\lambda}{\lambda_{x}^{\prime}} \frac{1}{\tan (\Psi)}=\frac{\lambda}{\lambda_{y}^{\prime}}$

Unter Berücksichtigung von GI. (5) und GI. (6) kann der Spiegelneigungswinkel damit durch Gl. (8) ermittelt werden.

$\varphi_{\mathrm{x}}=\frac{1}{4} \frac{\lambda}{\lambda_{y}^{\prime}} \tan (\Psi)=\frac{\lambda}{\lambda_{x}^{\prime}}$

Für die Auswertung kommen somit zwei grundlegende Strategien infrage. Zum einen ist es möglich, den Betrag des Spiegelneigungswinkels durch die Auswertung des Amplitudenspektrums von einem oder mehrerer Zeilensignale zu ermitteln (GI. (8) rechter Ausdruck). Diese Strategie wurde in der Literatur anhand anderer Interferometerkonfigurationen bereits verfolgt [6], [7]. Mit diesem Ansatz entfällt allerdings die Richtungsinformation der Spiegelneigung, weil nur der Betrag der Streifenbreite $\lambda_{x}^{\prime}$ ermittelt werden kann. Weiterhin ist auf Basis der bisherigen Untersuchungen davon auszugehen, dass mit diesem Ansatz nicht die gewünschte Auflösung erreicht werden kann.

Die zweite Möglichkeit besteht in der Auswertung der Spaltensignale. Die Streifenbreite $\lambda_{y}^{\prime}$ kann dabei über das Spektrum und der Winkel $\Psi$ über die Phasenverschiebung der einzelnen sinusförmigen Signale ermittelt werden.

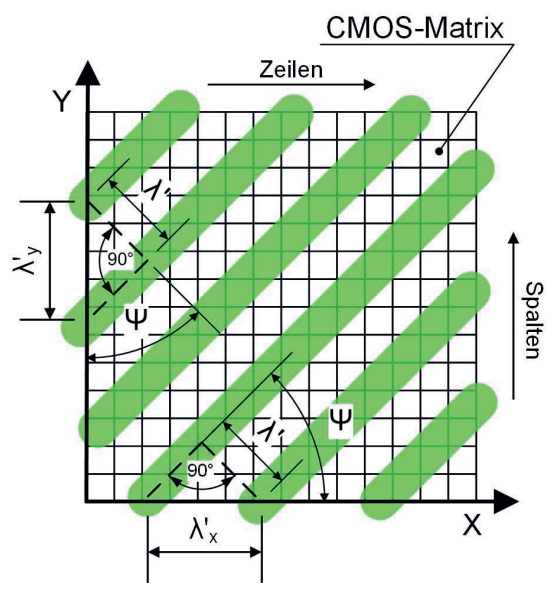

Abb. 4: Merkmale des Interferenzbildes

\section{Algorithmus}

Aufgrund der genannten Vorteile basiert der Algorithmus für die Auswertung des Interferenzbildes auf der Analyse der Spaltensignale (Abbildung 5).

Dazu wird von jeder Spalte der CMOS-Matrix das Amplituden- und Phasenspektrum mithilfe der schnellen Fourier-Transformation (FFT) ermittelt.

Das Amplitudenspektrum dieser Signale enthält außerhalb des Gleichanteils einen Peak. Über diesen wird die projizierte Streifenbreite $\lambda_{y}^{\prime}$ erfasst und über alle Spalten gemittelt.

Anschließend wird aus dem Phasenspektrum die zugehörige Phasenverschiebung $\varepsilon$ für jede Spalte ermittelt. Die in dem Datensatz auftretenden $2 \pi$-Phasensprünge werden anschließend korrigiert. Das ist möglich, weil bekannt ist, dass die Interferenzstreifen keine Unstetigkeitsstellen aufweisen. Mit der bereits ermittelten Wellenlänge $\lambda_{y}^{\prime}$ wird daraufhin die jeweilige Phasenverschiebung $\left[\varepsilon_{1}, \ldots, \varepsilon_{n}\right]$ der Sinussignale in eine Längenverschiebung $\left[Y_{1}\right.$, $\ldots, \mathrm{Y}_{\mathrm{n}}$ ] umgerechnet. In den so erhaltenen Datensatz wird eine Gerade durch eine Lineare Regression angepasst. Aus dem Anstieg der Geraden wird anschließend der gesuchte Winkel $\Psi$ berechnet.

Die eingesetzte Kamera besitzt eine Auflösung von 1024x1280 Pixel. Der für die ersten Versuche eingesetzte PC benötigte für die Auswertung des gesamten Interferenzbildes etwa 100 ms. Eine Reduktion der benötigten Zeit für die Auswertung ist unter Einsatz von FPGAs oder Grafikarten möglich.

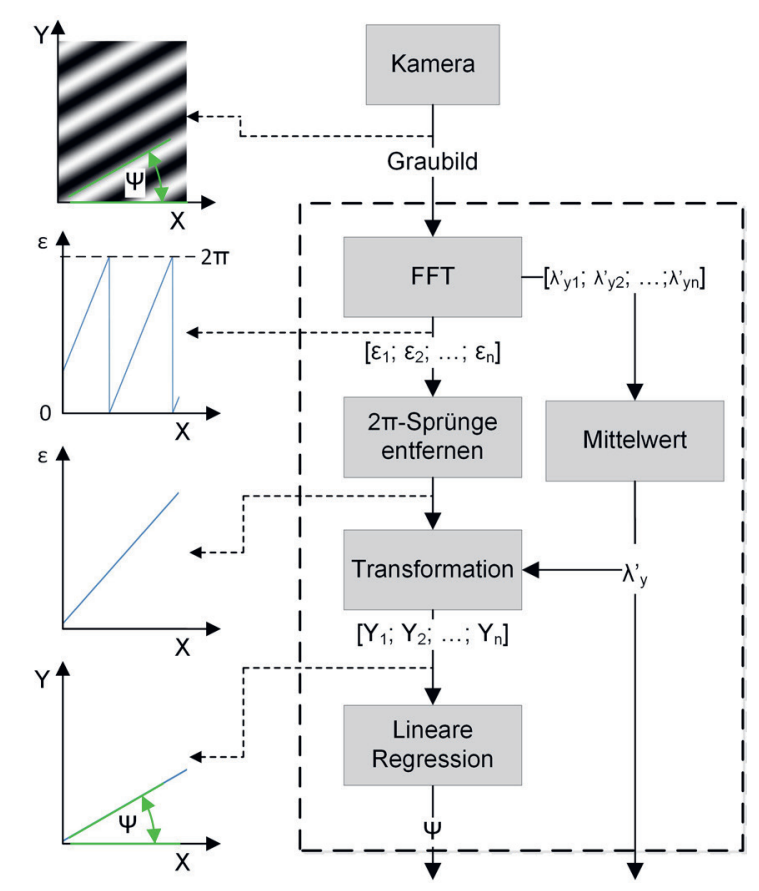

Abb. 5: Algorithmus zur Signalauswertung 


\section{Optischer Sensoraufbau}

Die Gültigkeit von GI. (8) beschränkt sich auf kollimiertes Licht und kann deshalb nicht auf den bisherigen Sensor angewandt werden (Abbildung 1). Bei diesem Sensor wird der Strahl senkrecht zum Strahlteiler in das Kösters-Prisma eingekoppelt $\left(\beta=30^{\circ}\right.$, Abbildung 6 ). Damit wird bewirkt, dass die Strahlen nach der Reflexion am Spiegel die jeweils gegenüberliegende Hälfte des Prismas durchlaufen. Unter Nutzung einer kurzkohärenten Strahlungsquelle konnte bei fünf getesteten Prismen nur in dieser Konfiguration eine Neigungsänderung der Interferenzstreifen beobachtet werden.

In Abbildung 7 sind die zu beobachtenden Interferenzbilder (Strahlenverlauf A) unter dem Einsatz einer kohärenten Strahlungsquelle (He$\mathrm{Ne}$ Laser) dargestellt. Bei kollimiertem Licht ist eine Neigungsänderung der Interferenzstreifen nicht zu beobachten und es treten in der Nullstellung des Spiegels (d.h. $\Psi_{x}=0$ ) keine sichtbaren Interferenzstreifen auf. Nach Gl. (7) kann das auf einen zu geringen Winkelversatz von $\Psi_{\text {y }}$ zurückgeführt werden, weil dieser in direktem Zusammenhang mit der Streifenbreite $\lambda^{\prime}$ y steht. Dieser Winkelversatz kann nur durch Abweichungen von der Idealform des Prismas erzeugt werden und ist eine notwendige Voraussetzung für die Realisierung des Sensorprinzips. Der Strahlenverlauf A weist eine hohe Symmetrie zwischen den Strahlen auf, wodurch dieser Winkelversatz minimiert wird.

Das Kösters-Prisma kann jedoch durch Variation des Winkels $\beta$ noch unter einem weiteren Strahlenverlauf genutzt werden (Strahlenverlauf B). Bei diesem werden die Strahlen nach der Reflexion am Spiegel in die Prismenhälfte reflektiert, aus der sie vorher ausgetreten sind. Dadurch werden die beiden Strahlen gegenüber dem Strahlenverlauf A jeweils nur an einer Prismenhälfte totalreflektiert und gebrochen. Weil davon auszugehen ist, dass beide Prismenhälften unterschiedliche Fertigungsabweichungen aufweisen, ist bei diesem Strahlenverlauf mit

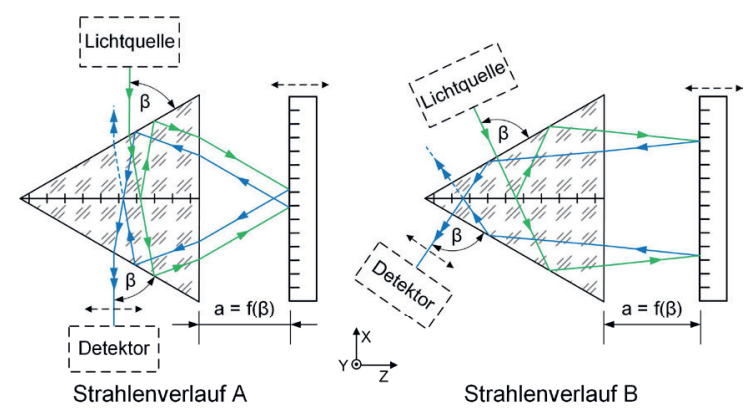

Abb. 6: Mögliche Strahlenverläufe einem größerem Winkelversatz $\Psi$ y zu rechnen. Diese Überlegung bestätigt die Beobachtung in Abbildung 7. Der Strahlenverlauf ist sowohl mit divergentem, als auch kollimiertem Licht nutzbar.

Gegenüber dem Strahlenverlauf $A$ ist dabei allerdings eine ausreichend kohärente Lichtquelle notwendig, denn durch die unterschiedliche Auslenkung der Strahlen ergeben sich geringfügig unterschiedliche Weglängen. Dadurch ist bei der Neigungsänderung des Spiegels neben der Rotation eine Translation der Interferenzstreifen zu beobachten. Dieser Effekt ist für die Funktionsweise des Sensorprinzips nicht relevant, wenn eine Lichtquelle mit einer ausreichenden kohärenzlänge eingesetzt wird.

Bei dem modifizierten Sensor wird der Strahlenverlauf B verwendet (Abbildung 8). Der Laserstrahl wird senkrecht zur Prismenoberfläche eingekoppelt $\left(\beta=90^{\circ}\right)$, weil dadurch die Position des Interferenzbildes unabhängig vom Abstand des Spiegels ortsfest bleibt. In dieser Anordnung beeinflusst der Spiegelabstand lediglich den erreichbaren Messbereich. Limitierend ist dabei der laterale Versatz der Lichtstrahlen durch die Reflexion an dem geneigten Spiegel (Abbildung 8). Eine Messung ist nicht mehr möglich, wenn dadurch die Lichtintensität auf der CMOS-Matrix zu gering wird. Dieser Effekt limitiert auch den Messbereich von AKFs.

Die Ausdehnung des Sensors wird primär durch das verwendete Prisma und den eingesetzten Kollimator vorgegeben.

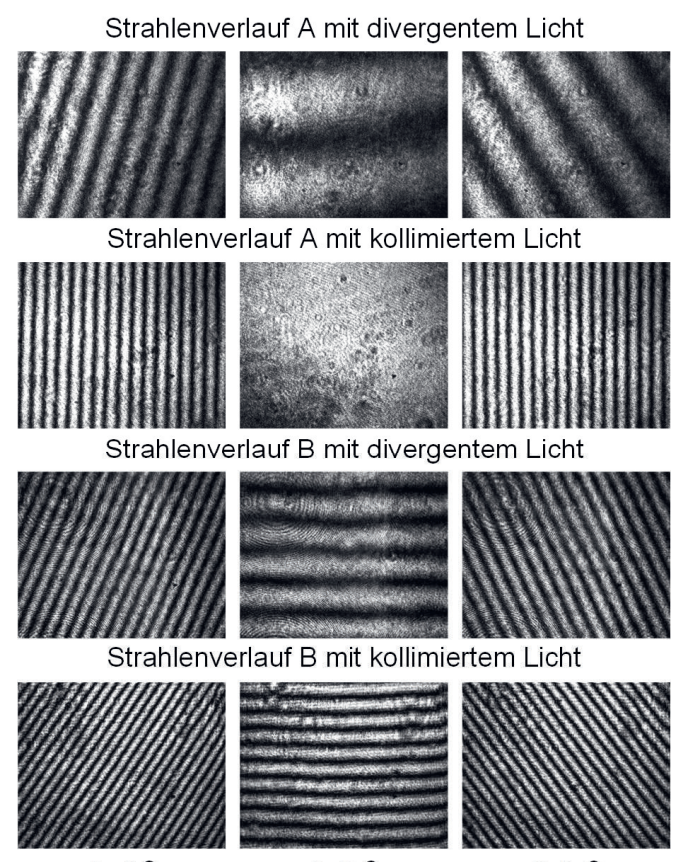

$\varphi_{\mathrm{x}}<0$

$\varphi_{x} \approx 0$

$\varphi_{\mathrm{x}}>0$

Abb. 7: Zu beobachtende Interferenzbilder 
Dabei wird die Größe des Kollimators durch den notwendigen Strahldurchmesser bestimmt. Dieser wiederum ergibt sich aus der Ausdehnung der aktiven Sensorfläche. An einem realisierten Prototyp kommt eine CMOSMatrix mit einer aktiven Sensorfläche von 6,66 $\mathrm{mm} \times 5,32 \mathrm{~mm}$ zum Einsatz. Der Kollimator hat eine Brennweite von $50 \mathrm{~mm}$. Die lange Kathete des verwendeten Kösters-Prismas beträgt 17 $\mathrm{mm}$. Damit ist die Baugröße des Sensors bereits deutlich kleiner als vergleichbare AKFs mit Brennweiten von $300 \mathrm{~mm}$ bis $1000 \mathrm{~mm}$. Eine weitere Reduzierung des benötigten Bauraums ist durch die Verwendung einer kleineren CMOS-Matrix möglich. Derartige Sensoren sind gegenwärtig bereits auf dem Markt erhältlich. Somit stellen die hier genannten Werte noch nicht die mit diesem Prinzip realisierbare Grenze dar [3].

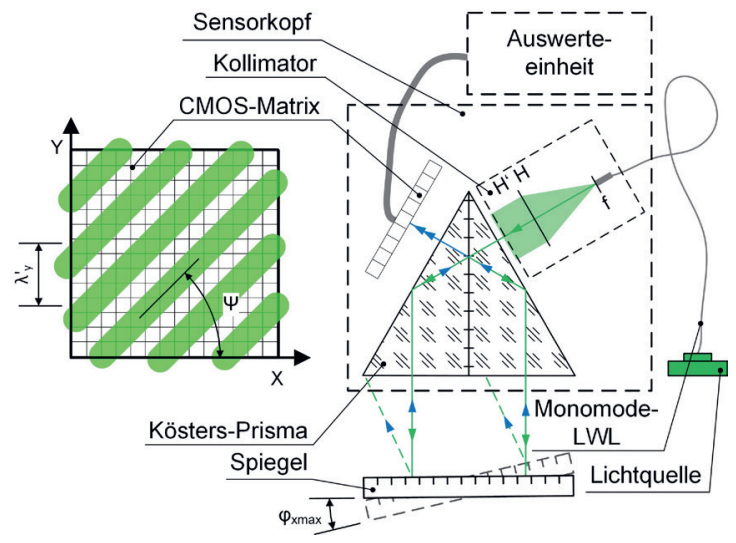

Abb. 8: Das überarbeitete Sensorkonzept zur absoluten Winkelmessung

\section{Messtechnische Untersuchungen}

Zur Überprüfung des Konzepts wurden erste Untersuchungen an einem einfachen Versuchsaufbau durchgeführt (Abbildung 9). Dabei wurde das neue Sensorkonzept mit einem kommerziell verfügbaren AKF verglichen. Das AKF besitzt eine Brennweite von $300 \mathrm{~mm}$.

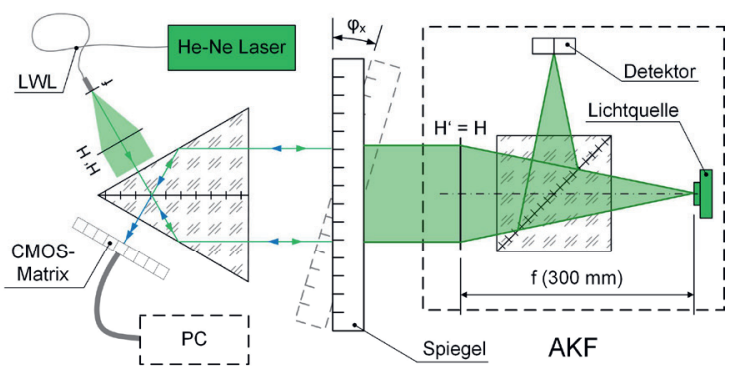

Abb. 9: Versuchsaufbau (nicht maßstabsgerecht)
Die eingesetzte Lichtquelle (He-Ne Laser) emittiert Licht mit einer Wellenlänge von 623,8 $\mathrm{nm}$. Zur Aufnahme einer Kennlinie wurde der Spiegel schrittweise manuell bewegt. Die Messwerte wurden jeweils zwischen zwei Schritten im stationären Zustand aufgenommen.

Gegenüber dem bisherigen Sensorkonzept wird zusätzlich die Streifenbreite erfasst. Ausgehend von GI. (2), GI. (3) und GI. (4) kann die Abhängigkeit der Streifenbreite $\lambda^{\prime}$ von dem Spiegelneigungswinkel und dem Neigungswinkel der Interferenzstreifen durch Gl. (9) und Gl. (10) beschrieben werden [3].

$$
\begin{aligned}
& \lambda^{\prime}=\frac{\lambda}{\sqrt{\Psi_{y}^{2}+\psi_{x}^{2}}}=\frac{\lambda}{\sqrt{\Psi_{y}^{2}+\left(4 \varphi_{x}\right)^{2}}} \\
& \lambda^{\prime}=\frac{\lambda}{\Psi_{y} \sqrt{1+\tan ^{2} \Psi}}
\end{aligned}
$$

In Abbildung 10 und 11 sind die gemessenen Abhängigkeiten dargestellt. Der Winkel $\varphi_{x}$ entspricht dem Referenzwert des AKFs. Zusätzlich enthalten beide Graphen die eigentlich gemessene Streifenbreite $\lambda_{y}$ '. Diese korreliert nach GI. (7) mit dem Winkelversatz $\Psi_{\mathrm{y}}$ und weist keine Abhängigkeit zu dem Neigungswinkel der Interferenzstreifen $\Psi$ und dem Spiegelneigungswinkel $\varphi_{\mathrm{x}}$ auf. In den Gleichungen (7), (9) und (10) ist der Winkelversatz $\Psi_{y}$ der einzige unbekannte Parameter. Anhand dieser Gleichungen und den Messwerten wurde der tatsächlich vorhandene Winkelversatz durch eine Regressionrechnung quantifiziert. Als Optimierungskriterium kam dabei die kleinste Summe der Fehlerquadrate zum Einsatz. Die Ergebnisse sind in Tabelle 1 enthalten.

Tab. 1: Die jeweiligen Ergebnisse der nichtlinearen Regression

\begin{tabular}{|c|c|c|c|}
\hline & GI. (7) & GI. (9) & GI. (10) \\
\hline$\Psi_{\text {y } \text { in “ }}$ & 263 & 263 & 282 \\
\hline$\Psi_{\text {y } \text { in mrad }}$ & 1,28 & 1,28 & 1,37 \\
\hline
\end{tabular}

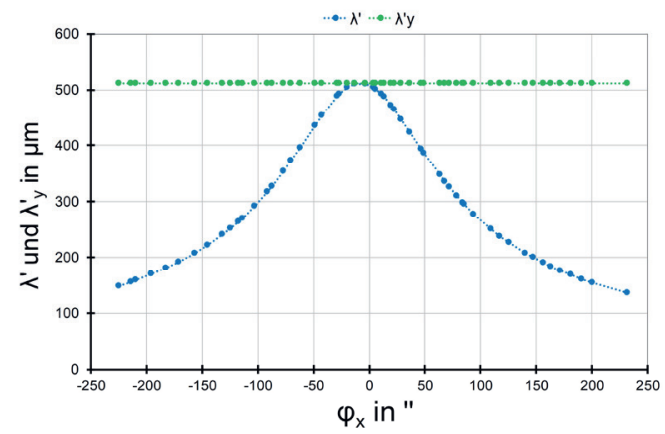

Abb. 10: Abhängigkeit zwischen der Streifenbreite der Interferenzstreifen und dem Spiegelneigungswinkel 


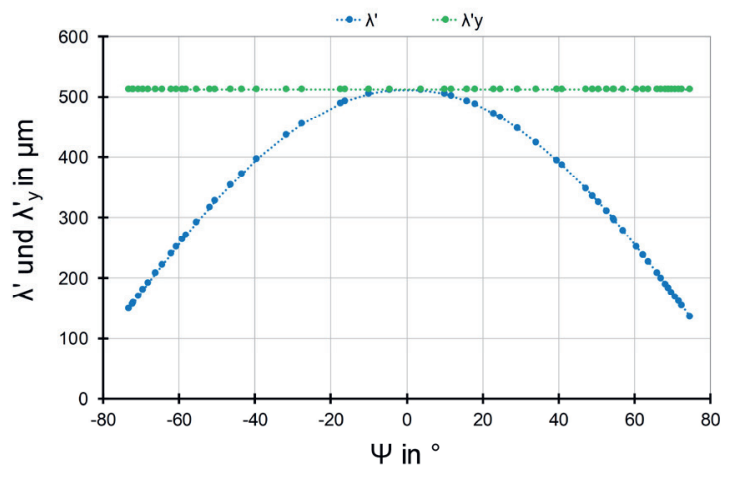

Abb. 11: Abhängigkeit zwischen der Streifenbreite und der Neigung der Interferenzstreifen

Für die eigentliche Messung des Winkels $\varphi_{x}$ wird GI. (8) angewandt. In Abbildung 12 ist die Differenz zwischen dem so ermittelten Spiegelneigungswinkel und dem Referenzwinkel des AKFs dargestellt. Es zeigt sich eine lineare Abweichung zwischen beiden Sensoren. Dafür können mehrere Gründe in Betracht gezogen werden. Ein wesentlicher Beitrag wird von dem Versuchsaufbau verursacht. Die Justierung entlang der optischen Achse beider Sensoren war nur bedingt möglich. Dadurch messen beide Sensoren nicht exakt den gleichen Winkel. Ein weiterer Beitrag kann der unzureichenden Information über die CMOS-Matrix zugeordnet werden. Diese bildet die Referenz für die Streifenbreite und den Neigungswinkel der Interferenzstreifen. Deshalb ist die Kenntnis über die Anordnung und Ausdehnung der einzelnen Pixel erforderlich. Im Rahmen dieser Untersuchungen wurden dafür die Angaben des Herstellers verwendet. Dieser gibt die Größe der Pixel mit 5,2 $\mu \mathrm{m}$ an. Diesem Wert ist jedoch keine Unsicherheit zugeordnet und somit unvollständig.

Für eine erste Einschätzung der Vergleichsmessung wurden deshalb die linearen Abweichungen korrigiert (Abbildung 13).

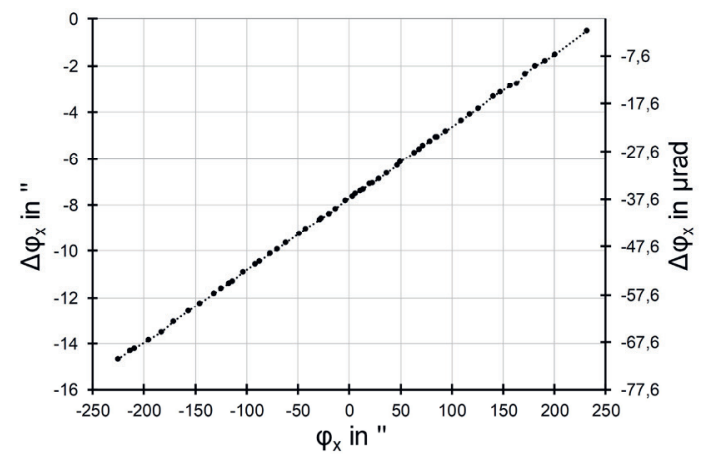

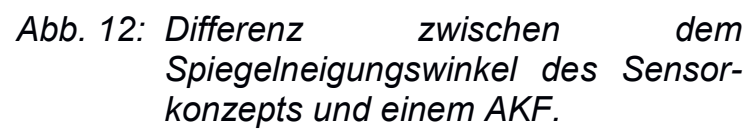

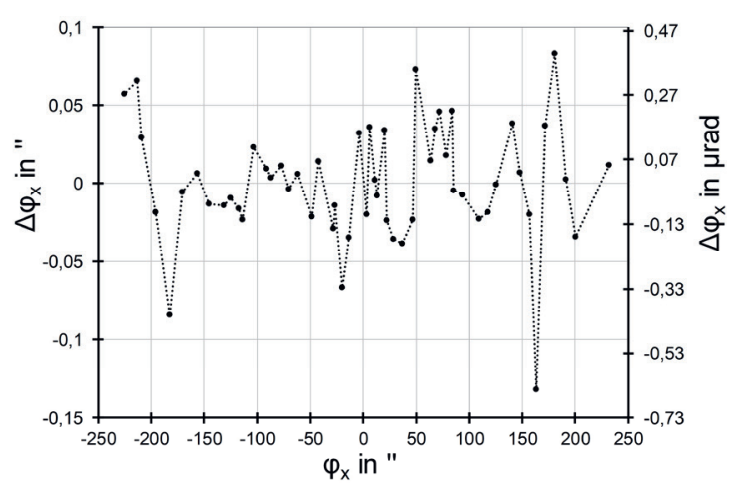

Abb. 13: Die Messwerte aus Abbildung 12 ohne lineare Abweichungen.

Ohne die linearen Abweichungen zeigen sich keine weiteren systematischen Abweichungen gegenüber GI. (8). Zwischen den Messwerten wurde der Versuchsaufbau durch die manuelle Änderung der Spiegelposition negativ beeinflusst. Für weiterführende Untersuchungen sind dementsprechend bessere Versuchsbedingungen notwendig.

In [2] wurde bereits eine Auflösung von unter 5 nrad $(0,001$ ") mit diesem Prinzip erreicht. Im Unterschied zu den Ergebnissen in [2] wurde in den ersten Versuchen mit dem modifizierten Aufbau zusätzlich ein Messbereich von 5 mrad (1000“) abgedeckt.

\section{Zusammenfassung}

Die Funktionsfähigkeit des Sensorkonzepts konnte anhand der ersten Messungen gezeigt werden. Für weiterführende Untersuchungen wurde ein Prototyp entworfen [3].

Gegenüber AKFs ermöglicht das Prinzip eine deutlich kleinere Bauweise bei gleichbleibender bzw. höherer Sensorauflösung. Über die Messunsicherheit kann zum gegenwärtigen Zeitpunkt noch keine Aussage getroffen werden.

\section{Literaturnachweis}

[1] V. Ullmann, E. Manske, DGaO-Proceedings, $118(2017)$

[2] V. Ullmann, E. Oertel, E. Manske, Meas. Sci. Technol. 29 (2018); doi:10.1088/13616501/aab252

[3] E. Oertel, TU IImenau, Masterarbeit (2018)

[4] M. Welter, TU IImenau, Dissertation (2005)

[5] J. Sindram, TU IImenau, Masterarbeit (2016)

[6] S. Strube, G. Molnar, H. Danzebrink, Meas. Sci. Technol. 22 (2011); doi:10.1088/09570233/22/9/094026

[7] G. Molnar, S. Strube, P. Köchert, H. Danzebrink, J. Flügge, Meas. Sci. Technol. 27 (2016); doi: 10.1088/0957-0233/27/8/084011 